\title{
Tesis electrónicas de la Universidad de Los Andes: adaptación y uso de la Plataforma TEDE
}

\author{
N. Fabiola Rosales \\ Master in Library and Information Science. Ingeniero de Sistemas. \\ E-mail: rfabiola@ula.ve \\ Marlene Bauste \\ Especialista en Gerencia de Servicios de Información. Geógrafa. \\ E-mail: bauste@ula.ve
}

\author{
Eliana Guzmán \\ Magister Scientiae en Modelado y Simulación de Sistemas. Magister \\ Scientiae en Ingeniería de Mantenimiento. Ingeniero de Sistemas. \\ E-mail: eliana@ula.ve \\ José Bianco \\ TSU en Informática. Universidad de Los Andes, Mérida, Venezuela. \\ E-mail: jbianco@ula.ve
}

\section{Resumen}

Uno de los proyectos de gran envergadura de la Universidad de Los Andes (ULA) es la creación de la Biblioteca Digital de Tesis Electrónicas. Su consecución garantiza elevados niveles de interacción entre los actores internos y externos de la ULA a través de la consulta y publicación de la producción científica e intelectual de la Institución.

En este artículo se presenta, en particular, la experiencia en la adaptación de la plataforma TEDE para la publicación de tesis electrónicas. La adaptación de la plataforma TEDE se llevó a cabo en tres etapas, a saber: a) Instalación, configuración y prueba de funcionalidad de la plataforma, b) Configuración y pruebas para el caso ULA, y c) Adaptación de la plataforma a la ULA. El resultado de esta adaptación fue una plataforma única que cumple con los requerimientos básicos establecidos para la publicación de tesis electrónicas en la ULA y que se ajusta perfectamente a cualquier institución académica de Venezuela. Muestra de esta experiencia ULA es su implantación en otras universidades nacionales como la Universidad del Zulia (LUZ), la Universidad Simón Bolívar (USB) y la Universidad Nacional Experimental de los Llanos Ezequiel Zamora (UNELLEZ).

\section{Palabras clave}

Tesis y disertaciones electrónicas. TEDE. Universidad de Los Andes. Venezuela. Software. Repositorio institucional. Adaptación. Software libre. Biblioteca digital.

\section{Teses eletrônicas da Universidade de Los Andes: adaptação e uso da Plataforma TEDE}

\section{Resumo}

Um dos projetos de grande envergadura da Universidad de los Andes é a criação de sua Biblioteca Digital de Teses Eletrônicas. Sua implantação garantirá altos níveis de interação entre agentes internos e externos à ULA, através da consulta e da publicação da produção científica e intelectual da instituição. Este artigo apresenta, em particular, a experiência da adaptação da plataforma TEDE para a publicação de teses eletrônicas. A adaptação da plataforma TEDE ocorreu em três etapas, a saber: a) Instalação, configuração e teste de funcionalidade da plataforma, b) Configuração e testes para o caso da ULA, e c) Adaptação da plataforma à ULA. O resultado da adaptação foi a implementação de uma plataforma única que cumpre os requisitos básicos estabelecidos para a publicação de teses eletrônicas na ULA e que se adequa perfeitamente a qualquer instituição acadêmica da Venezuela. Isso se comprova por sua implantação em outras universidades nacionais como a Universidad del Zulia (LUZ), a Universidad Simón Bolívar (USB) e a Universidad Nacional Experimental de los Llanos Ezequiel Zamora (UNELLEZ).

\section{Palavras-chave}

Teses e dissertações eletrônicas. TEDE. Universidad de Los Andes. Venezuela. Software. Repositório institucional. Adaptação. Software livre. Biblioteca digital.

\section{Universidad de Los Andes electronic thesis: adaptation and use of the TEDE Plataform}

\section{Abstract}

One of the projects of great importance of the University of the Andes (ULA) is the creation of the Digital Electronic Thesis Library. Its implementation guarantee high levels of interaction between the internal and external actors of the ULA through the consultation and publication of the intellectual production of the institution. This article presents the experience in the adaptation of the TEDE platform for electronic theses publication. The adaptation of the TEDE platform was carried out in three stages, that is to say: a) Installation, configuration and test of functionality of the platform, b) Configuration and tests for the ULA case, and c) Adaptation of the platform to the ULA. The result of this adaptation was a unique platform that fulfills the established basic requirements for the electronic thesis publication at the $U L A$, and that adjusts perfectly to any academic institution of Venezuela. An illustration of this ULA experience is the implementation of this recently created ULA platform in other national universities such as the University of Zulia (LUZ), Simón Bolivar University (USB), and the National and Experimental University of the Llanos Ezequiel Zamora (UNELLEZ).

\section{Keywords}

Electronic theses and dissertation. TEDE. Universidad de Los Andes. Venezuela. Software. Institutional repository. Adaptation. Open source. Digital library. 


\section{N. Fabiola Rosales / Marlene Bauste / Eliana Guzmán / José Bianco}

\section{INTRODUCCIÓN}

La producción y difusión de tesis y disertaciones en formato electrónico ha llegado a convertirse en un fenómeno mundial que ha motivado la creación de redes de conocimiento a distintas escalas, locales, nacionales e internacionales, ejemplo en Venezuela es la impulsada por la Asociación Nacional de Directores de Bibliotecas, Redes y Servicios de Información del Sector Académico, Universitario y de Investigación (ANABISAI).

La Universidad de Los Andes (ULA), representada en ANABISAI por los Servicios Bibliotecarios Universitarios (SERBIULA), pionero en el estudio de las plataformas para la publicación de tesis electrónicas llevó a cabo la evaluación de dos de las tres plataformas propuestas en el Proyecto de "La Red de Bibliotecas Digitales de Venezuela" con la finalidad de ofrecer a las instituciones participantes una guía que permitiera seleccionar la plataforma que mas se adaptara a sus necesidades y requerimientos. Del estudio realizado, la plataforma seleccionada por y para la ULA fue el sistema de Tesis y Disertaciones Electrónicas (TEDE), desarrollada por el Instituto Brasileño de Información en Ciencia y Tecnología (Ibict).

La ULA como universidad bicentenaria que es, la primera en producción científica y la tercera más importante en tamaño en Venezuela, cuenta con un importante repositorio de trabajos de grado, de ascenso, y de tesis y disertaciones, como resultado de su producción académica, de investigación y extensión. La necesidad de almacenar, difundir, preservar e intercambiar este legado intelectual es el propósito fundamental de SERBIULA para dar cumplimiento a la misión y objetivos universitarios.

Crear la Biblioteca Digital de Tesis Electrónicas que garantice elevados niveles de interacción entre los actores internos y externos de la ULA, a través de la consulta y publicación de la producción científica e intelectual de la Institución, es el proyecto de mayor envergadura que se está ejecutando en SERBIULA. Recientemente, la ULA por resolución emanada por el Consejo Universitario se acogió a la declaración de Berlín de la Iniciativa de Acceso Abierto (OAI) a la información y al conocimiento, lo que asegura la publicación libre de toda su producción científica e intelectual.

El objetivo de este artículo es presentar la experiencia en la adaptación de la plataforma TEDE, así como las recomendaciones a seguir por otras instituciones para la correspondiente adaptación de manera de garantizar el éxito en su funcionamiento.

\section{ANTECEDENTES}

En el año 1998 se presentó ante el Consejo Directivo de SERBIULA el anteproyecto de digitalización de trabajos de grado (memorias de pregrado) en búsqueda de dar solución a la consulta del ingente patrimonio científico e intelectual que reposaba en las estanterías de cada una de las bibliotecas que conformaban el sistema bibliotecario.

Las Facultades de Humanidades y Educación e Ingeniería sirvieron de modelo piloto para llevar adelante este trabajo. A nivel de los respectivos Consejos de Facultad se redefinieron las normas y procedimientos para el suministro de los trabajos de grado. A partir de ese momento se comenzó a recibir en estas Bibliotecas las versiones impresas y en formato electrónico - disquetes, CD-ROM y posteriormente DVD- de los trabajos.

Desde entonces, SERBIULA ha trabajado en el estudio de estándares y software para la publicación electrónica, así como en la revisión y actualización de los reglamentos para el suministro de trabajos de grado, en los programas de formación, capacitación y actualización del personal y de los usuarios.

En el mes de octubre de 2003, la Universidad de Los Andes a través de SERBIULA organizó las Jornadas de Actualización de la Biblioteca Universitaria: Las Bibliotecas Venezolanas en la Era Digital, donde se firmó el acuerdo para la creación del Sistema Nacional de Información Digital entre las universidades e institutos de investigación del País conformado por ocho instituciones (ULA, IVIC, UCV, UNA, UC, UCLA, UDO y URBE) (1).

En noviembre del mismo año se realizó una Asamblea Extraordinaria de ANABISAI en la Universidad de Oriente (UDO), Nucleo Cumaná, con el propósito de formular el proyecto "Red Nacional de Bibliotecas Digitales" el cual contempló dentro de sus objetivos la creación de repositorios institucionales de tesis electrónicas". Allí se crearon los grupos de trabajo para abordar las distintas áreas de desarrollo, a saber:

\section{El estudio de las plataformas existentes.}

2. La creación del modelo nacional de metadatos y el modelo de publicación para la integración local, nacional e internacional. 
3. La gestión de recursos financieros para formación de los recursos humanos

4. La asesoría y evaluación para la implantación y ejecución del proyecto en cada una de las instituciones participantes.

\section{El estudio y análisis de la Ley de Derechos de Autor.}

A la ULA, le correspondió trabajar en el grupo responsable del estudio de las plataformas existentes para la publicación electrónica de tesis. La ULA inicia en marzo del 2004 la evaluación documental de TEDE, ETDVT y Cyberthesis para el proyecto nacional bajo los siguientes criterios: Software libre para permitir la adaptación a las instituciones, interfase amigable y uso del protocolo de recolección de metadatos OAI-PMH.

\section{DESCRIPCIÓN DE LAS PLATAFORMAS EVALUADAS}

TEDE: Software desarrollado por el Instituto Brasileño de Información en Ciencia y Tecnología (IBCT) (4). El sistema TEDE automatiza los procedimientos de publicación electrónica de tesis y disertaciones. Este sistema tiene como premisa básica que una publicación debe efectuarse de forma cooperativa involucrando al autor, al curso de postgrado y a la biblioteca. Su instalación necesita de un sistema operativo basado en la plataforma UNIX/LINUX, además requiere de los siguientes software básicos: Apache como servidor Web, MySQL como gestor de base de datos y PHP para la creación de las páginas Web.

Este software cuenta con dos versiones, una modular y otra simplificada.

La versión modular involucra en el proceso de publicación de las tesis a los autores, unidades académicas y biblioteca. Es una plataforma que descentraliza el proceso de publicación. Posee un módulo que esta bajo la responsabilidad de los autores, así mismo existen otros módulos bajo la responsabilidad de la biblioteca, de las unidades académicas y del administrador de la plataforma. También lo integra un módulo de búsqueda.

La versión simplificada no posee los módulos para autores y unidades académicas. La publicación del electrónico se realiza desde el módulo que está bajo la responsabilidad de la biblioteca.

Cyberthesis: "Cybertesis es el resultado de un programa de cooperación entre la Universidad de Montreal, la
Universidad de Lyon2 y la Universidad de Chile, con el apoyo de Fonds Francophone des Inforoutes y Unesco"(2),(6). Utiliza scripts escritos en Java y que son interpretados por programas GNU-GPL que ejecutan instrucciones que generan los archivos finales de uso. Básicamente, el sistema toma una tesis escrita en MSWord, RTF o PDF, marcada con una plantilla desarrollada para identificar y tipificar las partes formales de una tesis, y la transforma en un archivo XML, desde el cual produce una versión HTML y PDF del documento completo y de cada una de sus partes. Todos los archivos producidos son almacenados dentro de un directorio creado para cada tesis.

La Cadena de Producción Cybertesis se divide en tres partes:

- La marcación de la tesis por parte del autor que está haciendo el documento

- La generación de documentos XML, HTML y PDF a partir del documento marcado

- La indexación de estos documentos usando un motor de recuperación para archivos XML

ETD-VT: Software desarrollado por el Virginia Tech (EE.UU). Es una plataforma que permite la publicación de las tesis y disertaciones en formato electrónico. Los requerimientos de software para esta plataforma son MySQL como gestor de base de datos, PERL como lenguaje de programación y CGI.pm (3).

Consta de 5 módulos: Módulo de consulta (busca y lista), Módulo de suministro de tesis, Módulo de administración (Control de usuarios, Mantenimiento, Publicación), Módulo de ayuda y Módulo para el manejo del protocolo OAI-PMH.

Del resultado de esta evaluación para el proyecto nacional se concluyó que estas plataformas pueden ser usadas y adaptadas por cualquiera de las instituciones participantes.

\section{CRITERIOS PARA LA SELECCIÓN DE LA PLATAFORMA A UTILIZAR EN ULA}

Para la selección de la plataforma a utilizar en la ULA se determinó que la misma debía cumplir con los siguientes requerimientos: 
- Software libre que cumpla con la licencia GNU-GPL, para garantizar la modificación del código fuente en el proceso de adaptación al modelo ULA.

- Interfaz usuario amigable y sencilla, con múltiples puntos de acceso para llegar al texto completo de la tesis, así como también la posibilidad de contacto con: autores, tutores o asesores, jurados e instituciones.

- Módulo de búsqueda y recuperación

- Módulo de suministro en línea para los autores.

- Módulo para suministro sin intervención de los actores.

- Módulo de administración del repositorio.

- Sistema de ayuda en todos los módulos.

- Control de acceso al sistema.

- Notificación automática a los autores vía correo electrónico.

- Modelo de Metadatos que considere, además de los elementos dados en el modelo ETD-MS (5) los siguientes:

- Datos del repositorio Institucional.

- Datos de la Biblioteca depositaria del impreso.

- Lugar de defensa, ya que la ULA posee núcleos en diferentes ciudades de Venezuela.

- Datos asociados a los autores como URL del currículo, correo electrónico y forma de ser citado.

- Datos asociados a los programas de postgrado y pregrado.

- Ente Financiador.

- Proveedor de metadatos vía protocolo OAI-PMH.

En base a los criterios antes mencionados, TEDE resultó ser la plataforma que mas se adaptaba a las necesidades planteadas.

\section{TEDE: ADAPTACIÓN}

La adaptación de la plataforma se inicia en el mes de abril de 2004, para ese momento no existía una versión en español. La metodología seguida se describe a continuación:

\section{Instalación, configuración y prueba de funcionalidad de la plataforma}

Los procesos de instalación y configuración se dan en una etapa inicial usando las fuentes originales, con la finalidad de probar el funcionamiento de la plataforma configurada para el caso Brasileño.

El proceso de instalación de la plataforma es rápido y sencillo. Aun, cuando se siguieron los pasos indicados en el manual, fue necesario realizar algunas modificaciones en el archivo de configuración del lenguaje PHP (php.ini), para el manejo de caracteres especiales y para el tamaño de archivos a transferir hacia el servidor.

\section{Configuración y pruebas para el caso ULA}

La plataforma debía adaptarse para la publicación de tesis de postgrado y para trabajos especiales desarrollados en el pregrado. Esto obligó a crear dos repositorios, uno para pregrado y otro para postgrado.

La configuración y pruebas se realizaron bajo la versión modular del software TEDE tomado en cuenta las siguientes consideraciones:

- Los términos que describen el área de conocimiento (materias/subject) están en portugués, los cuales deben ser traducidos al español para crear su propia descripción. Estos se encuentran en la base de datos asociada a la tabla "AreaConhecimento" (área de conocimiento).

- Los grados académicos para los postgrados contenidos en la tabla "Grau" deben ser adaptados a los otorgados por la Institución. En el caso de pregrado se usó el término "No aplica" ya que las instituciones (universidades venezolanas) otorgan a este nivel un título académico.

- Tutor, cotutores, jurados, entre otros, son roles asociados a una persona, se encuentran definidos en la tabla "Papel" y los mismos deben ser adecuados al nombre usado por la institución.

- Los tipos de materiales de apoyo están definidos en la tabla "TiposApoio" y deben ser modificados.

- La plataforma maneja una tabla para país (Pais) y otra para los estados (UnidadeFederativa), las mismas deben adaptarse a la división político administrativa del país. 
- Los campos CPF y CNPJ son equivalentes en Venezuela a la cédula de identidad y RIF respectivamente, por lo que deben usarse sin máscara.

- Traducir al español los archivos de configuración: tde_i18n/texto_es.txt, mensagens_es-SP, email_es-SP, tde_i18n/tde_admin/mensagens_es-SP, tde ${ }_{-}^{-}$i $18 \mathrm{n} /$ tde_aluno/ mensagens_es-SP, tde_i $1 \overline{1} 8 \mathrm{n} / \mathrm{tde}$ _biblioteca/ mensagens_es-SP, tde_i18n/tde_pos/mensagens_es-SP.

- Crear las ayudas en español y copiarlas en el directorio tde_ajuda/es-SP, guardando la misma estructura que tiene el directorio pt-BR.

- Sí, se cambia el nombre a la base de datos, se deben modificar los archivos "conexao.php" y "conexao fim.php", que se encuentran en el directorio "conexao".

Todas estas consideraciones no implicaron cambios en el software, sólo se aplicaron cambios a las tablas de la base de datos y a los archivos de configuración.

Las pruebas arrojaron que se debían hacer cambios en el código fuente ya que todas las variables que requieren traducción no se encontraban en los archivos de configuración y algunos procedimientos estaban validados sólo para el portugués, por ejemplo, para el caso de la variable psIdiomaPais sólo fue definida para tomar el valor pt-BR, y no el valor en español, que es es-SP. Otro ejemplo es el caso del Tutor, el cual se valida con el campo ppCod dado en la tabla Grau ( $\operatorname{ppCod}=3)$.

La versión en español que viene del software original requiere revisión de la traducción para la adaptación a la terminología usada en Venezuela. Es importante resaltar que al inicio de la evaluación del software se trabajó con la versión existente en portugués obligándonos a su traducción. Posteriormente, se libera la versión en español de TEDE lo que permitió comparar la traducción realizada con la ofrecida.

También se presentaron problemas en la incorporación del retrospectivo cuando se trató de usar la plataforma simplificada en conjunto con la modular, esto se debe a que fueron diseñadas para trabajar de manera independiente.

\section{Adaptación del software TEDE a la ULA}

Una vez estudiado y analizado el software TEDE se procedió a la modificación del código fuente y a la adaptación de las plataformas modular y simplificada. Como resultado de este procedimiento, se decidió integrar las dos plataformas, pues el uso de éstas por separado implicaba mayores cambios en el código fuente, además de mayor dedicación a la administración y mantenimiento de la base de datos. Con ello se logró un modelo integrado que permite el uso de la plataforma de manera práctica y transparente para el usuario final y para los administradores.

A continuación se muestra en los Cuadros 1 y 2 la estructura original del directorio de archivos del software TEDE en las versiones modular y simplificada.

\section{Estructura del software TEDE: Versión Integrada ULA}

Se procedió a la integración de las dos versiones del software TEDE para cumplir con la premisa establecida, que el software a utilizar en la ULA, debe garantizar la publicación de la producción intelectual en dos tiempos, real y retrospectivo, con y sin la intervención de los autores y unidades académicas.

\section{CUADRO 1}

Estructura del software Tede original: Vvrsión modular

\begin{tabular}{l|l|l|l}
\hline Bibliotecas & Tde_busca & Tde_oai & Index.php \\
\hline Conexao & Tde_i18n & Tde_pos & \\
\hline Tde_admin & Tde_layout1 & Tde_publicada & \\
\hline Tde_ajuda & Tde_layout2 & Tde_TESTE & \\
\hline Tde_aluno & Tde_layout3 & Tedesimplificado & \\
\hline Tde_arquivos & Tde_layoutTESTE & Templates & \\
\hline Tde_biblioteca & Tde_MARC & TemplatesTESTE & \\
\hline
\end{tabular}

\section{CUADRO 2}

Estructura del software Tede original: versión simplificada

\begin{tabular}{l|l|l}
\hline Bibliotecas & Tde_busca & Tde_publicada \\
\hline Conexao & Tde_i18n & Tde_TESTE \\
\hline Tde_admin & Tde_layout1 & Templates \\
\hline Tde_ajuda & Tde_layout2 & TemplatesTESTE \\
\hline Tde_aluno & Tde_layout3 & Index.php \\
\hline Tde_arquivos & Tde_layoutTESTE & \\
\hline Tde_biblioteca & Tde_oai & \\
\hline
\end{tabular}


El estudio de las estructuras de las dos versiones, modular y simplificada, mostradas en los Cuadros 1 y 2, determinó que el directorio tde_biblioteca de la versión simplificada debía incluirse con otro nombre en el directorio raíz de la versión modular, ya que permite la incorporación de un documento, obviando la intervención de los autores y de las unidades académicas.

El programa index.php fue modificado para enlazar el modulo simplificado, así mismo, algunos programas se modificaron en la parte de interfaz con el usuario.

A continuación se muestra en la Cuadro 3 la estructura del directorio de archivos adaptada a la ULA.

El resultado de esta adaptación fue una plataforma única que cumple con los requerimientos básicos establecidos para la publicación de tesis electrónicas en la Universidad de Los Andes y, se ajusta perfectamente a cualquier institución académica del país. Muestra de esta experiencia ULA es su implantación en otras universidades nacionales como la Universidad del Zulia (LUZ), la Universidad Simón Bolívar (USB) y la Universidad Nacional Experimental de los Llanos Ezequiel Zamora (UNELLEZ).

La plataforma adaptada y en funcionamiento para la publicación de tesis y disertaciones de los estudios de postgrado de la ULA puede visitarse en la dirección http:/ tesispost.serbi.ula.ve/ y para los trabajos de pregrado en http://tesispre.serbi.ula.ve/. Ambas versiones están disponibles para ser descargadas en http:// www.serbi.ula.ve/descarga_TEDEULA/.

\section{CONCLUSIONES}

- Desde el punto de vista de la organización de la institución y de los procedimientos para la publicación de tesis electrónicas, la plataforma TEDE ofrece soluciones para las distintas necesidades identificadas, como son la publicación centralizada a través de la biblioteca o desencentralizada por medio de todos los actores involucrados, es decir, autores, unidades académicas y biblioteca.

- Desde el punto de vista informático, se concluye que las plataformas modular y simplificada poseen una buena estructura lógica y un código limpio. Estas características facilitaron el proceso de adaptación para la ULA, cuyo resultado es la integración de estos dos sistemas en uno solo, que permite el uso de manera práctica y transparente para el usuario final y para los administradores.

- La versión en español que viene del software original requiere revisión de la traducción para adecuarla al castellano.

- La adaptación se ajusta perfectamente a cualquier institución académica del país. Muestra de esta experiencia ULA es su implantación en otras universidades nacionales como la Universidad del Zulia (LUZ), la Universidad Simón Bolívar (USB) y la Universidad Nacional Experimental de los Llanos Ezequiel Zamora (UNELLEZ).

- El correo electrónico, cédula de identidad y RIF son campos que deberían ser no obligatorios, ya que dificultan la incorporación del retrospectivo, por lo que debe considerarse en actualizaciones futuras del software.

- La creación de una comunidad de usuarios de software TEDE para Venezuela es uno de los retos que se presenta.

\section{REFERENCIAS}

1. ANABISAI. Proyecto de Red Nacional de Bibliotecas Digitales (versión preliminar). Disponível em: < http://www.anabisai.org.ve/>. Acesso em: 26 jun. 2006.

2. CYBERTHESIS. Disponível em: < http://www.cybertesis.cl//>. Acesso em: 30 jun. 2006.

3. ETD-DB: digital library and archives. Disponível em: $<\underline{\text { http:// }}$ scholar.lib.vt.edu/ETD-db/developer/download/>. Acesso em: 30 jun. 2006.

4. INSTITUTO BRASILEIRO DE INFORMAÇÃO EM CIÊNCIA E TECNOLOGIA - IBICT. Sistemas de Publicação Eletrônica de Teses e Dissertações (TEDE Modular e TEDE Simplificado). Disponível em:

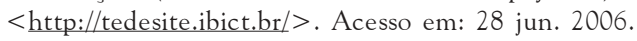

5. ETD-MS: an interoperability metadata standard for electronic theses and dissertations. Disponível em: < http://www.ndltd.org/ standards/metadata/current.html >. Acesso em: 20 jun. 2006.

6. UNESCO. Guide for electronic theses and dissertations. Disponível em:

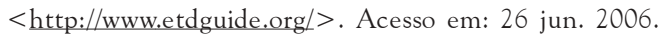

